\title{
PLANTEAMIENTOS FUNDAMENTALES DE LA RENOVACIÓN UNIVERSITARIA
}

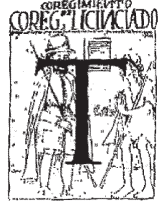

exto elaborado por la comisión ad-hoc creada por el Consejo Universitario de la Universidad Peruana Cayetano Heredia y organizada por los Drs. Mariano Querol y Leopoldo Chiappo. Se ha suprimido Universidad. todo lo relativo a aspectos internos de la

El contenido del documento representa la opinión conjunta y personal de los autores.

\section{INTEGRANTES:}

Dr. Mariano Querol
Dr. Renato Alarcón
Dr. Ramiro Castro de la Mata
Dr. Leopoldo Chiappo
Dr. Enrique Fernández
Ing. Hugo Pereyra

Dr. Rafael Acosta

Dr. Eduardo Barriga

Dr. Edmundo Beteta

Dr. Alberto Cazorla

Dr. Carlos Krumdieck

Dr. Hugo Lumbreras

Dr. Federico Moncloa

Dr. Carlos Monge

Sr. Víctor Puente Arnao

Dra. Susy Roedembeck

Dr. Fernando Samanez

Dr. Víctor Valdivia

Dr. Carlos Vidal

Ing. José Wittembury

Dr. Rafael Yockteng

\section{INTRODUCCIÓN}

Las páginas que siguen, constituyen el resultado de un esfuerzo común, orientado por aquello que sentimos como valores y dirigido hacia su plasmación en el ámbito universitario.

No somos un grupo de excepción, sino profesores y estudiantes universitarios con motivación ins- titucional, cohesionados por objetivos académicos trascendentes. Creemos tener en nuestras manos la responsabilidad de crear un nuevo concepto de la esencia de la universidad, cuyo sentido ha sido deformado por acontecimientos que, día a día, descalabran la cultura y las posibilidades de culturización en nuestra patria.

La Renovación Universitaria es un movimiento libre y solidario de profesores, estudiantes y exalumnos, dirigido a la superación y mejora continuas y permanentes, de las estructuras y los propósitos de la institución universitaria, de modo tal que se logren realizaciones culturales que redunden en beneficio de la comunidad social. El presente documento pretende esbozar los fundamentos ideológicos de esta concepción de universidad que, evidentemente, no es del todo original. Ella fue intuida por muchos desde el momento en que la Reforma Universitaria trastabilló, impotente para afrontar con una respuesta universitaria los problemas de la universidad y para aportar un enfoque universitario de los problemas de la colectividad.

El hecho histórico del origen del movimiento renovador en nuestra Universidad, no tiene precedentes por lo que significa de actitud colectiva. Desencadenado un clima intolerable en la Facultad de Medicina de la Universidad Nacional Mayor de San Marcos de Lima, se produjeron la renuncia de la mayoría de sus docentes y la creación de la Universidad Peruana Cayetano Heredia. Esta fue resultado de la decisión de actuar contra lo que consideramos radicales quiebras en la estructura institucional, así como de la necesidad de proseguir una labor universitaria auténtica. Reveló en primer término, consecuencia con elevados pronunciamientos principistas y en segundo lugar, capacidad para resistir a la presión política y para asumir riesgos económicos y hasta personales.

Por otra parte, puede afirmarse que la creación de Cayetano Heredia respondió al anhelo colectivo 
de poseer instituciones universitarias estables. Este anhelo así cristalizado, facilitó luego la ayuda del capital privado, hecho que se registraba por primera vez en América Latina.

E1 logro de una atmósfera auténticamente universitaria es expresión de la comunidad ideológica o generacional de un grupo de profesores, imbuído de la necesidad de luchar por los ideales universitarios, desvirtuados en la universidad "reformista", durante el período más crítico de su inveterada inestabilidad. Desde un punto de vista histórico, el grupo generacional ya existía en San Fernando. Él establece así una nueva escala de valores en la cual lo realmente académico pasa al primer plano. Es en Cayetano Heredia donde se toma conciencia de esta comunidad ideológica y se empieza a actuar en función de ella, tanto en forma individual como colectiva. Los estudiantes heredianos de la primera hora asumieron junto a sus profesores, los riesgos de la creación de una nueva universidad, identificados como aquéllos, con los principios de la Renovación.

En su actitud de vigilante autocrítica, la Renovación Universitaria sabe que la estabilidad y seriedad, contraparte del tumulto permanente de la llamada Reforma Universitaria, pueden ser sobrevaloradas por la colectividad y por la propia institución. El conformismo tendría así un apoyo en la posición favorable de la opinión pública del país y del extranjero. Es preciso luchar contra ese conformismo y buscar ideales absolutos y no comparativos con otras Universidades, para llevar a la Universidad Renovadora a siempre superados niveles de vida institucional.

\section{ESENCIAS Y MISIÓN DE LA UNIVERSIDAD}

Es difícil encontrar una proposición que exponga los caracteres genéricos y diferenciales del concepto de universidad, con suficiente claridad y exactitud. La esencia de la universidad, es decir su naturaleza, lo permanente e invariable en ella y lo que la distingue de otra cosa, puede ser considerada desde tres puntos de vista fundamentales: cultural, formativo y social.

\section{Esencia cultural de la universidad}

La universidad es un centro para la promoción de la cultura. Esto implica la conservación, la transmisión y el incremento del patrimonio cultural. Es innegable que, así considerada, la universidad ha de constituirse como la conjunción de un lugar geográfico, un grupo social y un factor temporal. En la universidad repercuten los cambios culturales universales; de ella emanan influencias, también inexorablemente universales, sobre la cultura. Es por ello que la universidad es una institución dinámica, en constante renovación, concorde con la realidad cultural y científica de cada momento histórico, al cual, a su vez, impone su sello humanístico.

\section{Esencia formativa de la universidad}

Al hablar de esencia de la universidad, en su aspecto formativo, debe hacerse hincapié en lo pertinente a la formación individual. En efecto, la universidad ha de formar a las personas de acuerdo a determinadas normas culturales y éticas, como un fin en sí, sin menoscabo de que aquéllas sirvan, directa o indirectamente, a la colectividad. En la universidad el hombre busca obtener una visión coherente de la realidad. Esta búsqueda puede tener fines culturales, objetivos prácticos o ambos a la vez. La universidad debe satisfacer estas dos exigencias que entrañan sendas misiones: una inmanente y teleológico; otra contingente y pragmática.

Esencial y teleológicamente, la universidad ha de cumplir una misión formativa, humanística, tendiente al enriquecimiento cultural y al desarrollo integrativo armónico del individuo, que conduzca a la adquisición $\mathrm{y}$ al manejo del conocimiento como fundamento de un estilo de vida que le haga profesar, esto es, vivir en libre y leal conformidad con las altas normas éticas que la universidad ofrece. Así el hombre, al par que 
enaltece su propia existencia con el conocimiento, se halla en condiciones de utilizarlo en forma mediata o inmediata, directa o indirecta, al servicio de la comunidad social. El arquetipo del individuo, formado en el ámbito de la esencia universitaria, es aquel que alcanza una forma de vida, impecable y plena, en un mundo que se esfuerza por abarcar con unción, merced a su visión sintetizadora y humanística.

La misión contingente de la universidad entraña una finalidad inmediata que si bien está contenida en la esencia, no la sobrepasa ni debe desvirtuarla. Dicha finalidad es de índole práctica, conformativa de actividades funcionales, fundamentalmente tecnificadas, asentadas sobre una visión analítica del mundo para determinado momento histórico. En este sentido la universidad se pone al servicio directo e inmediato de la colectividad.

La libre elección implica la libre aceptación, tácita o explícita, de los principios esenciales de la institución que el individuo ha escogido. El universitario debe identificarse con el sentido de la cultura que se imparte en la universidad y con los principios deontológicos sobre los que se afirma la actividad universitaria.

\section{Esencia social de la universidad}

La universidad constituye, desde el punto de vista social, una institución corporativa, sin fines de lucro, dimanada de la comunidad social, y por ende, al servicio de la misma. La esencia social de la universidad no debe ser considerada exclusivamente por la función de la institución en la comunidad, sino que la universidad es, en sí, un grupo social ya que, en su estructura constitutiva, se le podría definir como una corporación de maestros, alumnos y exalumnos, cuyo fin común es el afán, espontáneo e indeclinable, de aprender, enseñar e investigar. La universidad es una creación humana que depende de los hombres que la conforman. Estos, reunidos en grupo corporativo, han de sentirse portadores de un patrimonio cultural, deben estar imbuídos del valor esencial de dicho patrimonio y deben vivir en la convicción de su misión trascendente.

El análisis del aspecto social, interno, de la estructura universitaria conduce necesariamente a tratar sobre las características, la relación y el gobierno de los grupos que la conforman.

La coordinación jerárquica, la compenetración de los objetivos, la integración de los individuos dentro del grupo y de los grupos dentro de la corporación universitaria, el carácter esencialmente dinámico de todo ello y su posible y continua variabilidad, son factores de extrema importancia para el despliegue de las funciones de la corporación universitaria. En ella, el conocimiento integrativo, al par teórico y técnico, formativo y conformativo, humanístico y pragmático, sólo puede brotar, conservarse, trasmitirse y desarrollarse cuando las condiciones sociológicas son las adecuadas.

De lo dicho anteriormente se desprende que el gobierno de la universidad, debe surgir de lo que la institución es en sí, y ha de ser aquel que más convenga al mantenimiento de sus principios, al cumplimiento de su misión y a la realización exclusiva de sus fines. El tipo de gobierno de la corporación universitaria ha de ser pues, el resultante de la realidad esencial interna de la institución. Toda imposición que en este aspecto viniera por influencia de factores extrauniversitarios, constituye una aberración. De esto se infiere que el concepto de la autonomía universitaria no sólo es un principio sino que, antes y fundamentalmente, es inmanente a la esencia de la universidad.

La universidad sirve a la colectividad, en tanto que cumple con sus misiones cultural y formativa. Ambas están contenidas en la esencia de la universidad, ya que el ideal de la acción universitaria es promover la cultura en beneficio colectivo y formar individuos íntegros, con vuelo y altura teóricos, capacitados para el despliegue constructivo y con flexi- 
bilidad orientada hacia la realización de valores. En función de ello es que la universidad es una institución al servicio de la colectividad y que está abierta, en principio, a toda la comunidad social. La participación en la actividad universitaria es un derecho de todo individuo que posea las condiciones, éticas e intelectuales, para cumplir con las obligaciones que impone dicha actividad. En el seno de la corporación universitaria todos los integrantes habrán de cumplir y hacer cumplir las obligaciones dimanadas de la esencia misma de la institución.

En suma, la universidad es una institución corporativa, sin fines de lucro, dimanada de la comunidad social, conformada por maestros, alumnos y exalumnos, unidos en un afán espontáneo e indeclinable de aprender, enseñar e investigar. Constituye un centro formativo, humanístico y de servicio a la colectividad para la promoción de la cultura, autónomo en su gobierno y en constante renovación.

\section{PRINCIPIOS Y BASES DE LA UNIVERSIDAD}

Los principios, como enunciados normativos, constituyen las condiciones indispensables para el mantenimiento de la esencia y el cumplimiento de la misión de la universidad en determinado momento histórico. Deben fluir con toda la flexibilidad que supone su dependencia de la concepción que, sobre la esencia y misión de la universidad, se tenga en una situación histórica concreta. Son, por ende, revisables y susceptibles de renovada reflexión. De allí que todo planteamiento principista en materia universitaria deba, lógicamente, tener un carácter racional y no dogmático.

Las bases de organización posibilitan la eficacia operativa fáctica de los principios. Son válidas en tanto se adapten a las características y condiciones de cada institución universitaria.

En suma, los principios que se enuncien y las bases que se establezcan, son valiosos sólo en tanto que permitan y garanticen la actividad de la universidad acorde con su esencia y su misión.

\section{PRINCIPIOS}

1. Principio Cultural. La universidad debe mantener un alto nivel de vida institucional y académica que, siendo estable y ejemplar le permita desempeñar, activamente, su misión esencial de conservar, acrecentar y transmitir la cultura.

Aunque la universidad esté enmarcada dentro de determinados patrones, hábitos y condiciones culturales, no puede limitarse a significar un mero reflejo pasivo del contorno, sino, más bien, está llamada a ejercer una tarea activa de promoción y desarrollo de los niveles culturales en que actúa. En este sentido, la universidad cumple una función política, en cuanto la política se refiere al manejo técnico de !os asuntos comunes para el bienestar y desarrollo del conjunto.

Se considera como alto nivel de vida académica e institucional, aquel que se desenvuelve bajo rigurosas exigencias en lo que se refiere al cultivo de la ciencia y la práctica de los valores espirituales. La vida académica debe preservar la calidad de los conocimientos que se transmiten y amplían, procurando elevar el nivel de los educandos a los requerimientos de una enseñanza seria, por difícil que ella sea, pues la universidad debe preparar a la "élite" dirigente. Sólo cuando está garantizado un alto nivel académico puede la universidad cumplir el papel que le corresponde en la sociedad contemporánea:

a) Ser creativa en un universo de conocimientos científicos cada vez más complicados y especializados, lo cual implica que se lleven a cabo tareas de investigación. La universidad debe exigir a sus hombres el cumplimiento de estas tareas, como propósito fundamental de su misión cultural. No hacerlo equivaldría a desvirtuar sus objetivos, a cercenar el ámbito de sus posibilidades $\mathrm{y}$, lo que es peor, a perpetuar una nefasta tónica escolarizante. La investigación 
científica, como labor primordialmente universitaria es realmente una necesidad en los países llamados emergentes, negarla o mediatizarla significaría además la persistencia de una inferioridad que se sustenta hoy en la dependencia y subordinación permanentes ante las naciones líderes. Cabe interrogarse, por otra parte, respecto a la conveniencia de la investigación pura o de la aplicada que se confunde a veces con la actividad tecnológica. Creemos que ambas son indispensables. La primera, sin tener como mira inmediata el resultado práctico, prepara a la élite que creando ciencia propia, libere al país de una suerte de sutil colonialismo científico. La segunda contribuye a la solución de problemas nacionales en diversos terrenos. Y en ambas formas de investigación compete a la universidad, asumir función rectora. Porque la Universidad, como ha escrito Hutchins, puede dejar de enseñar pero no debe dejar de investigar.

b) Preparar profesionales capacitados para manejar los conocimientos obtenidos en el nivel de la ciencia actual. Esto entraña, para casi todos sus efectos, un criterio de selección en el más universitario sentido de la frase. El espejismo de la llamada popularización de la enseñanza consistió en rebajar la calidad de los conocimientos para difundirlos entre el mayor número so capa de convertir a todos en universitarios. El verdadero sentido democrático de la enseñanza universitaria estriba en ofrecer igualdad de oportunidad a todos los miembros de la comunidad y seleccionar a aquellos que reúnan los requisitos intelectuales y morales indispensables para cumplir con las exigencias de un alto nivel académico. A medida que la universidad abre sus puertas indiscriminadamente a la masa, el nivel académico se rebaja a tal punto que la institución ya deja de ser universidad, salvo en el nombre.

El principio cultural enunciado constituye un punto de partida de recuperación de la institución universitaria latinoamericana, fuertemente dañada por la llamada popularización de la enseñanza. No se trata de defender a una "elite" clasista, sino de preservar en la comunidad la existencia de una "elite" intelectual, cualquiera que sea la procedencia socioeconómica de sus integrantes. El alto nivel de vida institucional supone la práctica de aquellos valores humanos que garantizan honorabilidad y estilo selectos en quienes integran la corporación universitaria. No cabe alto nivel de vida institucional cuando se relaja la disciplina y se pierde el respeto a la persona humana.

La estabilidad se refiere a la existencia de solidez y continuidad en la organización y trabajo institucionales. Las bases organizativas deben contener aspectos que garanticen estabilidad, fundamento principista no sólo de buen funcionamiento sino de desarrollo y progreso de la institución. La estabilidad por ello, impide el estancamiento, facilita el dinamismo creativo y permite la renovación de la universidad. La inestabilidad institucional lleva a la rutina y al descenso de nivel, como lo demuestra el caso de aquellas universidades zarandeadas por conflictos circunstanciales propios de la institución o ajenos a ella.

El carácter de ejemplaridad académica e institucional permite a la universidad desempeñar un papel activo frente a la comunidad social. No se trata de que la universidad reproduzca miméticamente las condiciones de la sociedad de que dimana sino que constituya por sí misma, por su ejemplaridad, fuerza educativa promotora de una elevación del nivel de vida del conjunto. La universidad es uno de los centros genéticos más decisivos en el desarrollo social. No debe olvidarse que, en su misión formativa, la universidad prepara a los líderes profesionales de las futuras generaciones.

2. Principio humanístico. La universidad debe favorecer el desarrollo perfectivo del hombre. Es necesario distinguir entre la dirección o sentido de este desarrollo perfectivo y las condiciones que favorecen su cumplimiento.

Lo primero supone la delimitación de una antropología axiológica, orientada a delinear la figura 
ejemplar del hombre en cuanto tal, basada en los datos científicos y en la reflexión, filosófica. Ha sido siempre un problema, explícita o implícitamente planteado en todo grupo educativo, definir el tipo de hombre que se pretende formar. La universidad en este aspecto, debe evitar desde un punto de vista humanístico, la idea de "tipo" o de "formación" como moldeo. Nada más antiuniversitario que pretender crear un especimen estereotipado. La labor, en tanto que formadora debe ser "educativa", en el sentido de que el proceso educativo, en vez de moldeo extrínseco y rígido de un "tipo", consiste, por el contrario, en sacar afuera, suscitar, cultivar lo intrínseco y propiamente humano del educando. Ello obliga a definir qué es lo intrínsecamente humano del hombre. Sin ánimo de ser exhaustivas, pueden señalarse las siguientes características interdependientes: inteligencia racional, libertad de decidir y proyectar, sentido de responsabilidad, creatividad y estimativa rica en discernimiento axiológico, conducentes a la estructuración y realización de una tabla de valores que confieran sentido a la existencia personal. La universidad debe favorecer, entonces, el desarrollo integrativo armónico de todo esto, que constituye lo mejor en la estructura biopsíquica del animal humano.

Para el desarrollo perfectivo del hombre, en el ámbito universitario han de reunirse las siguientes condiciones: a) Respeto a la dignidad de la persona humana en cuanto tal, con el consiguiente rechazo de todo tipo de discriminación racial, económica, social e ideológica; b) Libre discusión de ideas y cultivo de la imparcialidad objetiva, lo que supone la superación de toda forma de pensamiento dogmático o sectario. El riguroso ejercicio del sentido crítico de objetividad requiere reflexión fundamental epistemológica, tolerancia ideológica, sustento fáctico y congruencia lógica del saber, todo lo cual permite adentrarse en los alcances y límites del conocimiento. Esto último preserva tanto de la especulación sin base como de un cientificismo estrecho, proclive a empobrecer la capacidad de experiencia humana de la vida; c) Educación integral, capaz de lograr una armónica formación física, científica y humanística de la persona.
3. Principio corporativo. La vida universitaria se fundamenta en la vigencia de una auténtica comunidad filosófica, guiada por la autoridad de los maestros y robustecida por la participación cooperativa de los estudiantes y de los graduados.

La "philía" o amistad comunitaria que aproxima a los hombres por el trato frecuente en el saber de las cosas exige, de sus cultores, la posesión y la preservación de altas cualidades éticas e intelectuales. De ello fluye que la potestad del gobierno institucional será ejercitada por quienes desempeñan la misión magisterial, en la medida de su probidad y de su competencia. La autoridad docente debe estar, pues, sensatamente condicionada por el ascendiente real y efectivo del maestro sobre los alumnos y por el acatamiento consciente de éstos hacia lo que aquél es y encarna.

A su vez, el sentido cooperativo de la participación estudiantil, en todos los niveles de la vida universitaria, respalda permanentemente el potencial renovador que es garantía del desarrollo y perfeccionamiento de la institución. En los consejos de gobierno, esquemas vitales de la comunidad universitaria, la participación estudiantil debe tener carácter petitorio, informativo y de colaboración estrecha que de ninguna manera deje margen al conflicto o pugna de fuerzas en el ejercicio de la autoridad. La estructuración jerárquica de la comunidad universitaria excluye el fundamento pretendidamente democrático del llamado cogobierno estudiantil. La fundamentación moral de la autoridad docente torna inoperante y más bien lesivo, el argumento de una esencial acción fiscalizadora del cogobierno.

Finalmente, los graduados, tanto por el nexo entrañable que los mantiene dentro de la corporación, cuanto por el libre despliegue de su formación integral, deben contribuir, con el acierto de su perspectiva y a través de representación adecuada en organismos de la institución, a la mejor realización del ideal universitario. 
El principio corporativo, en suma, sirve eficazmente a los requisitos y fines del principio cultural de la universidad; se sustenta, en cuanto expresión colectiva de esfuerzos individuales coherentes, en los alcances del principio humanístico; y da pie a una vinculación solidaria con la sociedad circundante, por el sentido trascendente de su estructuración interna.

4. Principio de servicio comunitario. La universidad debe ser agente del desarrollo colectivo y no un instrumento en manos de grupos extrauniversitarios. La misión de servicio a la comunidad emerge y se proyecta a partir de la institución en sí. Esto no excluye la colaboración que la universidad debe recibir y dar a otros grupos humanos.

La universidad sirve a la comunidad social por el hecho mismo de constituir una corporación en la que se despliegan y cumplen las misiones esenciales cultural y formativa. Este servicio a la comunidad está pues ínsito en la esencia misma de la universidad, y será tanto mayor cuanto en mayor grado cumpla la universidad con su misión. En efecto, el servicio es a toda la comunidad, en su más amplio sentido, desde el momento en que la institución selecciona a los más aptos del grupo humano dentro del cual actúa. La cultura es un bien accesible a través de sus cultores $\mathrm{y}$, si ellos han sido formados en la universidad con sentido humanístico de la vida, serán los mediadores entre aquélla y la comunidad social para los aspectos del servicio que la universidad presta. Es justamente por la formación humana en el más amplio sentido, que la universidad es una institución de servicio. El servicio comunitario que la universidad presta, sobrepasa la voluntad individual de cada uno de sus miembros. Esta misión de la universidad queda allende los intereses individuales de personas o grupos dentro de la corporación. Cualquier institución que lleve el nombre de universidad proyecta su acción en la comunidad. Si la institución es en esencia y no sólo en nombre, una universidad, cumplirá a plenitud su misión de servicio. En caso contrario, sólo servirá como un núcleo de descomposición social y cultural.
La universidad debe, por otra parte, desplegar actividades en beneficio de la colectividad tanto en sus aspectos de servicio directo cuanto de servicio indirecto a través de la educación de otros grupos. El primero es el caso de la organización de programas constructivos para el desarrollo colectivo. El segundo incluye ciclos o programas de enseñanza o adiestramiento que - en las más diversas ramas del saber, en los sentidos más varios y en todos los niveles-, ha de impartir la universidad para el bienestar de la colectividad.

5. Principio de autonomía universitaria. La universidad tiene el derecho y el deber de tomar decisiones y ha de poseer la capacidad para ejecutarlas en lo que concierne a su organización y al desarrollo de actividades tendientes al cumplimiento específico de su misión.

EI principio de autonomía tiene raíces que brotan desde los orígenes de la corporación universitaria en la edad media. Sin embargo, el prestigio tradicional de la independencia del claustro no es fundamento suficiente del principio de autonomía universitaria. Es necesario reflexionar renovadamente sobre su sustento racional, sus alcances y límites. Y ello se hace tanto más urgente cuanto que los factores emocionales anexos a una tradición han dado lugar a confusión en la concepción del principio muchas veces sujeto de una apelación abusiva y deformante.

El análisis de la autonomía universitaria permite descubrir las siguientes notas que la constituyen en cuanto tal:

a) Derecho y deber decisorios. Un agente - sea individual o colectivo- es autónomo en la medida en que tiene el derecho de tomar decisiones. Sin la potestad volitiva que define una conducta a seguir, aparece la dependencia o subordinación a otra instancia decisoria. En el caso de la universidad, los organismos de gobierno son las instancias a las que compete la decisión. El derecho decisorio no puede ser arbitrario sino que ha de fundamentarse en los estatutos 
y reglamentos. En ellos la institución va plasmando su esencia, su misión, su proyección y sus sistemas reguladores, normativos de una vida corporativa plena de sentido de los valores. La autonomía se viola en este nivel cuando aparecen influencias o factores extrauniversitarios recortando o desnaturalizando la decisión. Respecto del deber decisorio, el fundamento se encuentra en la exigencia ética de cumplir la esencia corporativa de la institución. Renunciar a este deber o suspender su ejercicio, por consideraciones extrauniversitarias, significa una violación de la autonomía desde dentro. Por ejemplo, la apelación al Poder Estatal para que defina la forma de gobierno de la universidad significa una claudicación del deber decisorio; correlativamente, la imposición estatal de la forma de gobierno conlleva el desconocimiento del derecho decisorio inherente a la autonomía.

b) Capacidad de ejecución. Cualquier interferencia en la realización de las decisiones del gobierno institucional significa frustrar la autonomía en lo que concierne a su capacidad ejecutiva. Los paros y capturas del local constituyen, en sí, violación de autonomía.

c) Ámbito de competencia. Es jurisdicción del derecho decisorio aquello que concierne a la organización de actividades dirigidas al cumplimiento de la misión universitaria. Precisada la misión de la universidad, en tanto que fluye de su esencia, las actividades que ella despliega y la forma de organización que adopta, para realizarlas adecuada y eficazmente deben ser decididas por la propia institución.

El análisis racional del principio de autonomía universitaria muestra claramente el modo y los alcances pertinentes de este principio cuya fecundidad reside en que al proteger a la universidad de toda forma de poder impositivo extrínseco, garantiza lo libertad del pensamiento, la iniciativa de la inteligencia racional y el desarrollo multiforme del saber y de la cultura. El ejercicio de la autonomía universitaria debe estar garantizado por el orden institucional normativo de un estado de derecho. La universidad regimentada por una superestructura estatal totalitaria que obliga a una doctrina, oficial, sucumbe en la infertilidad cultural. Lo dicho permite desenmascarar la frecuente tergiversación del principio de autonomía, cuando se apela a él para ampararse en una suerte de derecho de asilo y de impunidad frente a las leyes del Estado. Grupos facciosos que ocupan el local, incendian o destruyen libros y enseres de la institución cometen actos agresivos - desde el insulto, la calumnia y la difamación hasta el ataque físico o la franca complicidad con fuerzas subversivas contra el orden público- invocan la autonomía, como si la jurisdicción de ésta pudiese alcanzar a la protección o inmunidad de delincuentes comunes o políticos por el solo hecho de estar matriculados en una universidad. Se trata aquí, indudablemente, no sólo de una deformación de la autonomía sino de un abuso del símbolo que representa. Si se considera que la universidad es el grupo social fundamentado por excelencia en la libertad, se justifica la tradicional respetabilidad que aquélla posee frente al Poder, mas no el tendencioso enfrentamiento permanente de dos "soberanías" en pugna. Hacerse acreedor del respeto público, reflejo de una bien entendida autonomía universitaria, es deber que la institución tiene que cumplir para realizar cabalmente su misión.

En suma, podemos decir con el Rector de la Universidad Nacional Autónoma de México, que hay violación de la autonomía cuando el Estado, por cualquier medio, coarta la independencia académica de la universidad o impide que ella se rija internamente; pero también existe cuando una corporación privada, un partido político, un grupo y en general, cualquier entidad o fuerza externa interviene en la vida de la universidad, sea alterándola, dificultando el cumplimiento de sus tareas o limitando de un modo o de otro las libertades que la sustentan.

6. Principio de renovación. La ideología, estructura, organización y funcionamiento de la universidad han de estar en continua revisión crítica y proyección creadora. 
Ellas han de brotar del despliegue de la autenticidad académica y del sentido de responsabilidad histórica de los integrantes de la corporación universitaria, en el pleno ejercicio de su libertad en tanto que tales. Sólo la continua renovación permite la adecuación de la universidad a las condiciones histórico-culturales; así, al evitar el anquilosamiento institucional, logra que la corporación viva libre de los desquiciamientos y de las crisis que, cada cierto tiempo, convulsionan a las universidades que no se renuevan.

La Universidad es el arquetipo de la institución cuya acción trasciende el presente y se proyecta hacia el futuro. En este sentido sigue siendo válida la definición que, hace más de cuatro siglos daba León Pinelo, según la cual la universidad es, fundamentalmente, su misión, el servicio que de ella se espera, lo que "debe ser". Este continuo cambio, esta renovación ínsitos en la esencia institucional, sólo pueden dimanar de movimientos esencialmente universitarios, surgidos del estudio sopesado y técnico, de los problemas que se presentan en la marcha corporativa. Por otra parte, la actitud renovadora en los planteamientos principistas, exige superar tanto el énfasis afectivo que ha dado lugar a los mitos reformistas, cuanto la solemnidad y rigidez de las proposiciones conservadoras. Ello no implica desconocer y dejar de aprovechar tanto lo que de racionalmente renovador tiene la intención reformista cuanto lo que de preser- vación de la estabilidad institucional, pudiese significar la actitud conservadora.

El impulso renovador se asienta sobre la visión de lo que la universidad ha de ser en el futuro. Aquellos movimientos universitarios que aparecen en función y al servicio exclusivo de lo que se pretende que la universidad sea en un presente, no conllevan trascendencia creadora y se esfuman rápidamente. Los intentos de modificar la universidad, para satisfacer intereses personales y rendir culto al dogmatismo son, en su esencia, pseudouniversitarios pues, aunque se originen dentro de la institución y aboquen a los fines propuestos, sus realizaciones son intrascendentes $y$, aun cuando sean efímeras, traban la renovación universitaria. Los cambios que desde el exterior se pretenda imponer a la universidad, lesionan la esencia institucional y mellan su autonomía. Por ello, pese a que puedan ser operantes, deforman su marcha a un grado tal que siempre impiden el cumplimiento de su misión.

El principio de renovación, emanado de una toma de conciencia de lo que se entiende por universidad, promueve los cambios favorables a la marcha institucional. Es ajeno a intereses pseudo o extrauniversitarios, que impiden que la institución se renueve a sí misma, en función de sus propias realidades, merced a su continuidad y sin menoscabo de su autonomía. 